\title{
Hyperimmune immunoglobulin for people with COVID-19 (Protocol)
}

Kimber C, Valk SJ, Chai KL, Piechotta V, Iannizzi C, Monsef I, Wood EM, Lamikanra AA, Roberts DJ, McQuilten Z, So-Osman C, Estcourt LJ, Skoetz N

Kimber C, Valk SJ, Chai KL, Piechotta V, Iannizzi C, Monsef I, Wood EM, Lamikanra AA, Roberts DJ, McQuilten Z, So-Osman C, Estcourt LJ, Skoetz N.

Hyperimmune immunoglobulin for people with COVID-19 (Protocol).

Cochrane Database of Systematic Reviews 2021, Issue 10. Art. No.: CD015167.

DOI: 10.1002/14651858.CD015167.

www.cochranelibrary.com 
TABLE OF CONTENTS

HEADER 1

ABSTRACT

BACKGROUND

OBJECTIVES

METHODS

ACKNOWLEDGEMENTS

REFERENCES

ADDITIONAL TABLES

APPENDICES

HISTORY

CONTRIBUTIONS OF AUTHORS

DECLARATIONS OF INTEREST

SOURCES OF SUPPORT

NOTES 
[Intervention Protocol]

\section{Hyperimmune immunoglobulin for people with COVID-19}

Catherine Kimber $1 a$, Sarah J Valk²b, Khai Li Chai ${ }^{3 c}$, Vanessa Piechotta ${ }^{4}$, Claire lannizzi ${ }^{5}$, Ina Monsef6, Erica M Wood ${ }^{3}$, Abigail A Lamikanra7, David J Roberts 8 , Zoe McQuilten³, Cynthia So-Osman' 9 , Lise J Estcourt10, Nicole Skoetz11

1UK. ${ }^{2}$ Center for Clinical Transfusion Research/Clinical Epidemiology, Sanquin/Leiden University Medical Center, Leiden, Netherlands. ${ }^{3}$ Transfusion Research Unit, School of Public Health and Preventive Medicine, Monash University, Melbourne, Australia. ${ }^{4}$ Department I of Internal Medicine, Center for Integrated Oncology Aachen Bonn Cologne Duesseldorf, Cochrane Haematology, Faculty of Medicine and University Hospital Cologne, University of Cologne, Cologne, Germany. ${ }^{5}$ Cologne, Germany. ${ }^{6}$ Faculty of Medicine and University Hospital Cologne, University of Cologne, Department I of Internal Medicine, Center for Integrated Oncology Aachen Bonn Cologne Duesseldorf, Cochrane Haematology, Cologne, Germany. ${ }^{7}$ Clinical, Research and Development, NHS Blood and Transplant, Oxford, UK. ${ }^{8}$ Systematic Review Initiative, NHS Blood and Transplant, Oxford, UK. 95anquin Blood Bank, Erasmus Medical Center, Amsterdam, Netherlands. 10Haematology/Transfusion Medicine, NHS Blood and Transplant, Oxford, UK. 11Faculty of Medicine and University Hospital Cologne, Department of Internal Medicine, Center for Integrated Oncology, University of Cologne, Cologne, Germany

${ }^{a}$ contributed equally. ${ }^{b}$ contributed equally. ${ }^{c}$ contributed equally

Contact address: Nicole Skoetz, nicole.skoetz@uk-koeln.de.

Editorial group: Cochrane Haematology Group.

Publication status and date: New, published in Issue 10, 2021.

Citation: Kimber C, Valk SJ, Chai KL, Piechotta V, Iannizzi C, Monsef I, Wood EM, Lamikanra AA, Roberts DJ, McQuilten Z, So-Osman C, Estcourt LJ, Skoetz N. Hyperimmune immunoglobulin for people with COVID-19 (Protocol). Cochrane Database of Systematic Reviews 2021, Issue 10. Art. No.: CD015167. DOI: 10.1002/14651858.CD015167.

Copyright @ 2021 The Cochrane Collaboration. Published by John Wiley \& Sons, Ltd.

\section{A B S T R A C T}

\section{Objectives}

This is a protocol for a Cochrane Review (intervention). The objectives are as follows:

Using a living systematic review approach, to assess whether hyperimmune immunoglobulin therapy is effective and safe in the treatment of people with COVID-19; and to maintain the currency of the evidence. 


\section{B A C K G R O U N D}

\section{Description of the condition}

This review was previously part of a parent review addressing convalescent plasma and hyperimmune intravenous immunoglobulins (hereafter referred to as hyperimmune immunoglobulins or hIVIG) for people with COVID-19 (Piechotta 2021). The review has been split to address hIVIG and convalescent plasma separately. Therefore, parts of this background text are shared between the two reviews. Specific adaptations related to hyperimmune immunoglobulin have been made.

The clinical syndrome coronavirus disease 2019 (COVID-19) is a new, rapidly emerging zoonotic infectious disease caused by severe acute respiratory syndrome coronavirus 2 (SARS-CoV-2; WHO 2020a). On 22 March 2020, the World Health Organization (WHO) declared the current COVID-19 outbreak to be a pandemic, with the outbreak resulting in more than 181 million confirmed cases and over 3.9 million deaths worldwide (WHO 2020b; WHO 2021a). Although there are similarities with historic coronavirus epidemics, with severe acute respiratory syndrome (SARS) and Middle East respiratory syndrome (MERS) responsible for 813 and 858 deaths respectively, the scale and impact of the COVID-19 pandemic presents unprecedented challenges to health facilities and healthcare workers all over the world (WHO 2007; WHO 2019).

Approximately $5 \%$ of people with COVID-19, and $20 \%$ of those hospitalised, experience severe disease requiring intensive care (Wiersinga 2020). Early reports suggested case fatality rates between $0.7 \%$ and $4 \%$ (WHO 2020a; WHO 2020c). More recent reports estimate wide-ranging case fatality rates, as low as $0.0 \%$ in Singapore and up to $9.0 \%$ in Mexico (Johns Hopkins 2021). However, these numbers should be interpreted with great care due to testing availability, underreporting of cases, and delays from confirmation of a case to time of death (Kim 2020), and factors associated with ethnicity, underlying health conditions, access to health care, and socioeconomic status (Williamson 2020).

The median incubation period of SARS-CoV-2 was reported to be five days, with $97.5 \%$ of cases developing symptoms within 11.5 days of infection (Lauer 2020). Common signs and symptoms can include fever, dry cough, fatigue, and sputum production (WHO 2020a). Post-viral olfactory dysfunction is reported in $5 \%$ to $85 \%$ of cases, with loss of both smell and taste reported (IzquierdoDominguez 2020). Other less commonly reported signs and symptoms are shortness of breath, sore throat, headache, myalgia or arthralgia, chills, nausea or vomiting, nasal congestion, diarrhoea, haemoptysis, and conjunctival congestion (WHO 2020a). Of the reported cases, $80 \%$ are estimated to have a mild or asymptomatic course of infection, and an estimated $5 \%$ of cases are admitted to an intensive care unit (ICU) with acute respiratory distress syndrome (ARDS), septic shock, multiple organ failure, or all three conditions (NCPERE 2020; WHO 2020a). A risk factor for developing infection and progressing to severe disease is old age, with people aged over 80 years at the highest risk of mortality. Other risk factors are cardiovascular disease, obesity, hypertension, diabetes, chronic respiratory disease, cancer, and compromised immune status (Chen 2020a; Huang 2020; Liang 2020; WHO 2020a; Wu 2020a). Early reports have suggested that people who are immune-compromised may not have an increased risk of being hospitalised with severe COVID-19 symptoms (D'Antiga 2020). However, evidence has been conflicting, with people with malignancy and recipients of solid organ and allogeneic stem cell transplants reported to have an increased risk of severe COVID-19 disease (Fung 2020; Sharma 2021).

There are indications that the SARS-CoV-2 virus is capable of inducing an excessive immune reaction in the host, with highly activated but decreased numbers of $T$ cells detected in the blood of people with COVID-19 (Xu 2020a). Early reports also showed that people critically ill with COVID-19 frequently exhibit an increased tendency to form blood clots, vascular inflammation, and elevated coagulation markers known as a hypercoagulable state (Maatman 2020). This hypercoagulable state is hypothesised to lead to the high burden of thromboembolic events seen in this population, with pulmonary embolism being the most common (Driggin 2020).

SARS-CoV-2 can infect people by binding to the angiotensinconverting enzyme 2 (ACE2), which functions as the viral receptor. ACE2 facilitates the entry of SARS-CoV-2 into the host cell and is most abundant on type II alveolar cells in the lungs (Tolouian 2020; Van de Veerdonk 2020).

\section{Description of the intervention}

Hyperimmune immunoglobulin (hIVIG) has been used to treat infections when no vaccine or pharmacological intervention is available. hIVIG is made from pools of human or animal donor serum with high neutralising titres (da Costa 2021). These hyperimmune sera contain polyclonal antibodies, which are concentrates of heterologous immunoglobulins, formed by intact IgG (immunoglobulin G, one of the five major classes of immunoglobulins) molecules or antigen-binding antibody fragments (da Costa 2021).

hIVIG provides passive immunisation with a level of therapeutic antibody that is more concentrated than convalescent plasma alone. Following an infection, antibodies are produced within B cell lineages exposed to antigens from the virus. These cells then produce immunoglobulins specific to different viral components. Preparations of hIVIG can be extracted from large amounts of pooled convalescent plasma, collected from people previously infected or vaccinated against the virus. Plasma may be obtained by separation of whole blood or by plasmapheresis. Alternatively, viral-specific antibodies can be injected with an adjuvant into a humanised animal modified to generate antibodies similar to those made naturally in humans.

Plasma from either source is then fractionated or extracted and purified to obtain the hIVIG preparation. Measures are then undertaken for viral inactivation or removal, which can include pasteurisation, solvent or detergent and low $\mathrm{pH}$ incubation and filtration. Regulatory oversight stipulates minimum viral reduction steps in the manufacturing process, which vary across countries (Bloch 2021). In 2020, a group of large plasma industry companies formed a plasma alliance to work together to develop hIVIG to be used in the treatment of COVID-19 (Farrugia 2020).

There is conflicting evidence about the effect of hIVIG for treating severe acute respiratory infections. Studies investigating the effectiveness of hIVIG for influenza have been contradictory, with at least one randomised controlled trial (RCT) showing effectiveness (Hung 2013), whereas another shows no benefit (Davey 2019). hIVIG 
has also been used in treatment of coronaviruses (including SARSCoV-1 and MERS-CoV) (da Costa 2021). In a systematic review, hIVIG has been found to be effective against cytomegalovirus in solid organ recipients (Bonaros 2008).

Apart from use in treatment of infections, hIVIG is also used to prevent infections in high-risk individuals or used as pre-exposure or post-exposure prophylaxis; for example, for varicella-zoster virus (PHE 2019).

A potential benefit of hIVIG over monoclonal antibody therapy is the diversity of antibodies obtained from a pool of donors. hIVIG may provide a wider range of specificity than monoclonal antibodies, be more effective in the setting of emerging variants, and provide a wide range of antiviral actions at relatively cheaper costs (Vandeberg 2021).

Benefits of hIVIG in comparison to convalescent plasma include lower volume, a higher concentration of antibody titre, the possibility of administration as an intramuscular injection (instead of intravenous infusion), and more convenience in storage and shipping conditions, allowing for more ease of transport (Bloch 2021). When compared to convalescent plasma, hIVIG also has the advantage of preventing the transfer of potentially harmful coagulation factors that are present in plasma products. The amount and antibody concentration can be more accurately dosed compared to convalescent plasma, and hyperimmune immunoglobulin can be prepared in a consistent manner (Hung 2013).

Not many studies have reported on adverse events of hIVIG but the safety profile of standard intravenous immunoglobulin is known, and the adverse events reported here are also likely to occur in hIVIG therapy. These include: infusion site pain; swelling and erythema; and immediate systemic reactions, such as head and body aches, chills, and fever (Stiehm 2013). Other, less common, early adverse reactions to immunoglobulin therapy are pulmonary complications, such as pulmonary embolism, pulmonary oedema, and pleural effusion, with transfusion-related acute lung injury (TRALI) also reported (Baudel 2020; Stiehm 2013). Anaphylactic and anaphylactoid reactions to immunoglobulin therapy are rare (Brennan 2003; Stiehm 2013). Delayed adverse events of immunoglobulin therapy, which occur within hours to days of initiation of immunoglobulin therapy, are persistent headaches (common), aseptic meningitis, renal failure, thromboembolic events, and haemolytic reactions (Sekul 1994; Stiehm 2013). Transmission of infectious agents has been described after administration of intravenous immunoglobulin, but this risk is considered to be low (Stiehm 2013). Other severe adverse events that occur late after administration are lung disease, enteritis, and dermatological disorders (Stiehm 2013).

A theoretical risk related to virus-specific antibodies, which are transferred with hIVIG administration, is an antibody-dependent enhancement of infection (Morens 1994). Here, virus-binding antibodies facilitate the entry and replication of virus particles into monocytes, macrophages, and granulocytic cells, and thereby increase the risk of more severe disease in the infected host. Antibody-dependent enhancement has not been demonstrated in people who have recovered and become reinfected with COVID-19, and there have been no reports of antibody-dependent enhancement in studies on monoclonal antibodies, convalescent plasma, or following COVID-19 vaccination. However, antibody- dependent enhancement has been seen with previous coronavirus infections when the antibodies given targeted a different serotype of the virus (Wan 2020; Wang 2014). The circulation of COVID-19 variants could increase the risk of antibody-dependent enhancement when the intervention contains antibodies targeting parts of the virus that are different from the original strain. Antibody-dependent enhancement is therefore a potentially harmful consequence of hIVIG therapy for COVID-19.

Further definitions of the terms used in this description can be found in the glossary of abbreviations and medical terms (Appendix 1).

In summary, the benefits of the intervention should be carefully considered in view of the risks of adverse events.

\section{How the intervention might work}

Hyperimmune immunoglobulin contains pathogen-specific neutralising antibodies, which can neutralise viral particles, and treatment with hIVIG confers passive immunity to recipients. The duration of conferred protection can differ depending on the timing of administration, ranging from weeks to months after treatment (Casadevall 2020).

By neutralising SARS-CoV-2 particles, early treatment with convalescent plasma, and by extrapolation hIVIG, is postulated to increase the individual's own capacity to clear the initial infection (Casadevall 2020; Robbins 1995). This could lead to a reduction in mortality and fewer hospitalised people progressing to the $I C U$, thus helping to lift pressure from global healthcare systems and increasing ICU capacity.

Preliminary evidence in humans and rhesus macaques has shown that reinfection with SARS-CoV-2 is not likely, with most (but not all) individuals who recovered from COVID-19 producing sufficient amounts of neutralising antibodies to protect against reinfection (Bao 2020; Wu 2020b). This implies that hIVIG made from convalescent plasma of people who have recovered from SARS-CoV-2 infection may be capable of conferring passive immunity. Retrospective studies also observed a potential correlation between the level of antibody titres in convalescent plasma and recovery after treatment (Joyner 2021; Shen 2020). It is important to note, however, that research in other coronavirus species has shown that immunity may not be long-lasting, with two to three years of protection estimated from work with SARS and MERS (Mo 2006; Payne 2016). Furthermore, there are indications that the severity of infection has an impact on antibody titres, with less severe disease leading to lower neutralising antibody response in people with SARS and COVID-19 (Ho 2005; Zhao 2020a). And, it is unclear exactly how often reinfection occurs, with the burden of reinfection likely to be underestimated, while at the same time a number of case reports of severe reinfection have been published (Iwasaki 2021).

\section{Why it is important to do this review}

There is a clear, urgent need for more information to guide clinical decision-making for people with COVID-19. Pharmacological treatment options are being investigated in many ongoing trials: currently, only treatment of dexamethasone and tocilizumab is proven to be effective in reducing mortality (Ghosn 2021; Horby 2020), and remdesivir and REGN-COV2 (the brand name of Regeneron Pharmaceuticals antibody cocktail Casirivimab/ 
imdevimab) are shown to reduce time to recovery (Beigel 2020; Horby 2021). The current treatment further consists of supportive care with extracorporeal membrane oxygenation in severe cases and oxygen supply in less severe cases (CDC 2020; WHO 2020d; WHO 2020f). Despite these treatments, people hospitalised with COVID-19 are still at a high risk of mortality. hIVIG could potentially be used alongside these treatments in ambulatory or hospitalised settings, if it proves to be effective and safe.

While our recent systematic review showed that convalescent plasma for the treatment of moderate to severe COVID-19 does not reduce mortality and has little to no impact on measures of clinical improvement (Piechotta 2021), we remain very uncertain about the efficacy of convalescent plasma in people with asymptomatic or mild disease. Therefore, it is important to continue to assess the possible effect of hIVIG and other antibody therapies on people with COVID-19, particularly in the early phase, and their potential role in specific subgroups of individuals.

Mass vaccination programmes have been underway since late 2020 (WHO 2020b). Until these vaccines are globally distributed, hIVIG may be a potential therapy for people with COVID-19. Even with effective vaccines, not everyone can be effectively vaccinated; for example, people who are temporarily or permanently immunecompromised, and very young children. hIVIG can be prepared and made available when enough potential donors have recovered from the infection, using readily available materials and methods (Bloch 2020). However, its safety and efficacy are not wellcharacterised, and there are costs associated with pursuing the use of hIVIG for treatment of COVID-19.

Several clinical trials investigating the safety and effectiveness of hIVIG have been announced, and their results will need to be interpreted with care. Thus, there needs to be a thorough understanding of the current body of evidence regarding the use of hIVIG for people with COVID-19, and an extensive review of the available literature is required.

\section{O B JECT IVES}

Using a living systematic review approach, to assess whether hyperimmune immunoglobulin therapy is effective and safe in the treatment of people with COVID-19; and to maintain the currency of the evidence.

\section{MET HOD S}

\section{Criteria for considering studies for this review}

\section{Types of studies}

The main description of methods is based on the standard template of the Cochrane Haematology review group and is in line with the parent review of this series, addressing convalescent plasma and hyperimmune immunoglobulins for people with COVID-19 (Piechotta 2021). We made specific adaptations related to the research question, we updated the methods slightly in light of the evolving research knowledge.

To assess the effectiveness and safety of hyperimmune immunoglobulins for the treatment of COVID-19, we will include RCTs, as such studies, if performed appropriately, give the best evidence for experimental therapies in highly controlled therapeutic settings. For RCT data, we will use the methods recommended by the Cochrane Handbook for Systematic Reviews of Interventions (Higgins 2021a, hereafter referred to as the Cochrane Handbook), as specified in the description of the methods. If we identify non-standard RCT designs, such as cluster-randomised trials and cross-over trials, we plan to include those, and apply the methods recommended in Chapter 23 of the Cochrane Handbook (Higgins 2021b). We will consider only the results from the first cycle of cross-over RCTs.

We will include full-text publications, pre-print articles, abstract publications, and results published in trials registries, if sufficient information is available on study design, characteristics of participants, interventions, and outcomes. We will not apply any limitation with respect to the length of follow-up.

\section{Types of participants}

We will include individuals with a suspected or confirmed diagnosis of COVID-19, with no age, gender, or ethnicity restrictions.

We will include trials that included participants with any disease severity. We will perform separate analyses for populations with ambulatory mild disease and for hospitalised participants with moderate to severe disease, according to the latest WHO clinical progression score (see Table 1; WHO 2020e).

We will exclude studies including populations with other coronavirus diseases (SARS or MERS). We will also exclude studies of populations with mixed viral diseases (e.g. influenza) unless the trial authors provide subgroup data for people with COVID-19.

\section{Types of interventions}

We will include the following interventions.

- Human hyperimmune immunoglobulin therapy.

- Hyperimmune animal sera containing polyclonal antibodies.

These include polyclonal immunoglobulin therapies containing full-length antibodies or fragment antibodies, and may be sourced from convalescent humans or immunised animals (including bovine, equine, rabbit, chicken, or other animal sources).

We will not include studies on: standard immunoglobulin (from non-convalescent donors) except as comparator; monoclonal antibodies; nanobodies; and microbodies.

We will not include studies of hIVIG used in healthy individuals to prevent COVID-19.

We will include the following comparisons for studies with a control arm.

- Hyperimmune immunoglobulin therapy versus control treatment; for example, drug treatments (including but not limited to hydroxychloroquine and remdesivir). Cointerventions are allowed but must be comparable between intervention groups.

- Hyperimmune immunoglobulin therapy versus standard care or placebo (i.e. saline solution).

\section{Types of outcome measures}

We evaluated core outcomes, as predefined by the Core Outcome Measures in Effectiveness Trials (COMET) Initiative for people 
with COVID-19 (COMET 2020), and additional outcomes that have been prioritised by consumer representatives, referees of previous versions of this review (Piechotta 2021), and the German guideline panel for inpatient therapy of people with COVID-19.

We defined outcome sets for two populations: individuals with a confirmed diagnosis of COVID-19 and moderate to severe disease, and individuals with a confirmed diagnosis of SARS-CoV-2 infection and asymptomatic or mild disease, according to the WHO clinical progression scale (WHO 2020e).

\section{Primary outcomes}

These critical outcomes will be included in the summary of findings table.

\section{Individuals with a confirmed diagnosis of COVID-19 and moderate to} severe disease

- All-cause mortality at day 28 , day 60 , time-to-event, and at hospital discharge

- Clinical status, at day 28 , day 60 , and up to longest follow-up, including the following:

* Worsening of clinical status: participants with clinical deterioration (new need for invasive mechanical ventilation) or death.

* Improvement of clinical status: participants discharged alive. Participants should be discharged without clinical deterioration or death.

- Quality of life, including fatigue and neurological status, assessed with standardised scales (e.g. WHOQOL-100, a standardised scale for assessing quality of life) at up to 7 days, up to 28 days, and longest follow-up available

- Adverse events (any grade, grade 1-2, grade 3-4), defined as the number of participants with any event and including potential relationship between intervention and adverse reaction (e.g. TRALI, transfusion-transmitted infection, transfusionassociated circulatory overload (TACO), transfusion-associated dyspnoea (TAD), acute transfusion reactions, headache, thromboembolic events)

- Serious adverse events, defined as the number of participants with any event

\section{Individuals with a confirmed diagnosis of SARS-CoV-2 infection and} asymptomatic or mild disease

- All-cause mortality at day 28 , day 60 , time-to-event, and at longest follow-up

- Admission to hospital or death within 28 days

- Symptom resolution:

* All initial symptoms resolved (asymptomatic) at day 14, day 28 , and up to longest follow-up

* Duration to symptom resolution

- Quality of life, including fatigue and neurological status, assessed with standardised scales (e.g. WHOQOL-100) at up to 7 days, up to 28 days, and longest follow-up available

- Adverse events (any grade, grade 1-2, grade 3-4), defined as the number of participants with any event and including the potential relationship between intervention and adverse reaction (e.g. TRALI, transfusion-transmitted infection, TACO, $T A D$, acute transfusion reactions, headache, thromboembolic events)
- Serious adverse events, defined as the number of participants with any event

\section{Secondary outcomes}

These important outcomes will not be included in the summary of findings table.

\section{Individuals with a confirmed diagnosis of COVID-19 and moderate to severe disease}

- Clinical status at day 28 , day 60 , and up to longest follow-up, including:

* Worsening of clinical status:

$\square$ New need for invasive mechanical ventilation;

$\square$ New need for non-invasive mechanical ventilation or high flow;

New need for oxygen by mask or nasal prongs.

* Improvement of clinical status:

$\square$ Weaning or liberation from invasive mechanical ventilation in surviving patients;

$\square$ Ventilator free days;

$\square$ Duration to liberation from invasive mechanical ventilation;

$\square$ Liberation from supplemental oxygen in surviving patients;

$\square$ Duration to liberation from supplemental oxygen.

- Need for dialysis at up to 28 days

- Admission to the intensive care unit (ICU) on day 28

- Duration of hospitalisation

- Viral clearance, assessed with reverse transcription polymerase chain reaction (RT-PCR) test for SARS-CoV-2 at baseline, up to 3, 7 , and 14 days

Individuals with a confirmed diagnosis of SARS-CoV-2 infection and asymptomatic or mild disease

- Clinical status at day 28 and up to longest follow-up, including:

* Worsening of clinical status (moderate to severe COVID-19 symptoms):

$\square$ Need for invasive mechanical ventilation;

$\square$ Need for non-invasive mechanical ventilation or high flow;

$\square$ Need for hospitalisation with the need for oxygen by mask or nasal prongs;

$\square$ Need for hospitalisation without oxygen therapy.

- Viral clearance, assessed with RT-PCR for SARS-CoV-2 at baseline, up to 3,7 , and 14 days

\section{Timing of outcome measurement}

For time-to-event outcomes, such as mortality, discharge from hospital, and improvement of clinical symptoms, we will include outcome measures representing the longest follow-up time available.

We will include all other outcome categories for the observational periods that the study publications report. We will include those adverse events occurring during active treatment, and long-term adverse events as well. If sufficient data are available, we plan to group the measurement time points of eligible outcomes - for example, adverse events and serious adverse events - into those measured directly after treatment (up to 7 days after treatment), 
medium-term outcomes (15 days after treatment) and longer-term outcomes (over 30 days after treatment).

\section{Search methods for identification of studies}

\section{Electronic searches}

We will search electronic databases according to methods suggested in the Cochrane Handbook (Lefebvre 2021). Studies reported in all languages are eligible, in order to limit language bias. If studies are published in languages other than those our review team can accommodate (English, Dutch, German, French, Italian, Malay, and Spanish), we will involve Cochrane Task Exchange to identify people within Cochrane to translate these studies.

As publication bias might influence all subsequent analyses and conclusions, we will search all potentially relevant trials registries in detail to detect ongoing as well as completed, but not yet published, studies. If outcome data are not available elsewhere, we will extract any outcome data found in the trial registry entry.

We will search the following databases and sources from 1 January 2019.

\section{Databases of medical literature}

- MEDLINE (Ovid, from 1 January 2019; Appendix 2)

- Embase (Ovid, from 1 January 2019; Appendix 3)

- Cochrane COVID-19 Study Register (covid-19.cochrane.org; from inception; Appendix 4)*

- PubMed (for e-publications ahead of print only; from 1 January 2019; Appendix 5)

- World Health Organization COVID-19 Global literature on coronavirus disease (https://search.bvsalud.org/globalliterature-on-novel-coronavirus-2019-ncov/; from inception) without references of MEDLINE and PubMed; Appendix 6)

- Epistemonikos, LOVE List Coronavirus disease (COVID-19) (app.iloveevidence.com/loves; from inception; Appendix 7)

*The Cochrane COVID-19 Study Register is a specialised register built within the Cochrane Register of Studies (CRS) and is maintained by Cochrane Information Specialists. Complete data sources and search methods for the register are available at: community.cochrane.org/about-covid-19-study-register. The register contains study reports from several sources, including:

- daily searches of PubMed;

- daily searches of ClinicalTrials.gov;

- weekly searches of Embase.com;

- weekly searches of the WHO International Clinical Trials Registry Platform (ICTRP);

- monthly searches of the Cochrane Central Register of Controlled Trials (CENTRAL).

\section{Living systematic review considerations}

We will carry out weekly searches for completed and ongoing studies. We will check weekly for newly emerging hyperimmune immunoglobulins, and review search methods and strategies approximately monthly, to ensure they reflect any terminology changes in the topic area, or in the databases. We will adapt the strategy where necessary.

\section{Searching other resources}

We will handsearch the reference lists of all identified studies. We will also contact experts in the field, drug manufacturers, and regulatory agencies in order to retrieve information on unpublished studies.

\section{Living systematic review considerations}

The signal for updating this review will stem from weekly monitoring of the published relevant RCTs via the database search, as described under "Electronic searches". Once the decision to update the review has been made, the methods mentioned in this section will be incorporated in the review update.

\section{Data collection and analysis}

\section{Selection of studies}

Using Covidence software, two review authors (from among SJV, $\mathrm{KLC}, \mathrm{VP}, \mathrm{CK}, \mathrm{Cl}$, and NS) will independently screen for eligibility the results of the search strategies, by reading the abstracts. We will code the abstracts as either 'retrieve' or 'do not retrieve'. In the case of disagreement, or if it is unclear whether we should retrieve the abstract or not, we will obtain the full-text publication for further discussion. Two review authors will assess the full-text articles of selected studies. If the two review authors are unable to reach a consensus, we will consult a third review author to reach a final decision.

We will document the study selection process in a flow chart, as recommended in the PRISMA statement (Moher 2009), and show the total numbers of retrieved references and the numbers of included and excluded studies. We will list all studies excluded after full-text assessment, and the reasons for their exclusion, in the Characteristics of excluded studies table.

\section{Living systematic review considerations}

Two review authors will screen records derived from weekly searches to identify new studies.

\section{Data extraction and management}

Two review authors (from among SJV, KLC, VP, CK, and $\mathrm{Cl}$ ) will independently assess eligible studies obtained in the process of study selection (as described above) for methodological quality and risk of bias. If the review authors are unable to reach a consensus, we will consult a third review author to reach a final decision.

Two review authors (from among SJV, $\mathrm{KLC}, \mathrm{CK}, \mathrm{Cl}$, and VP) will extract data using a customised data extraction form, developed in Microsoft Excel (Microsoft Corporation 2018). Another review author (Cl, VP, or NS) will verify the accuracy and (where applicable) the plausibility of extractions and assessment. We will conduct data extraction according to the guidelines proposed by Cochrane ( $\mathrm{Li}$ 2021). If the review authors are unable to reach a consensus, we will consult a third review author. All extracted data will be summarised in tables or appendices.

We will collate multiple reports of one study so that the study, and not the report, is the unit of analysis.

We will extract the following information. 
- General information: author, title, source, publication date, country, language, duplicate publications.

- Quality assessment: study design, confounding, definition of risk estimates, bias arising from the randomisation process: due to deviations from the intended interventions; due to missing outcome data; in measurement of the outcome; and in selection of the reported results.

- Study characteristics: trial design, setting and dates, source of participants, inclusion/exclusion criteria, comparability of groups, treatment cross-overs, compliance with assigned treatment, length of follow-up.

- Participant characteristics: age, gender, ethnicity, number of participants recruited/allocated/evaluated, disease, severity of disease, additional diagnoses, baseline serostatus, previous treatments (e.g. experimental drug therapies, oxygen therapy, ventilation), whether the donors were tested by nasal swabs or whether the plasma was tested.

- Interventions: hyperimmune immunoglobulin therapy, concomitant therapy, duration of follow-up, donors' disease severity, methods of hyperimmune immunoglobulin preparation, whether hyperimmune immunoglobulin dosage was adjusted based on batch-dependent neutralising antibody levels.

* For studies including a control group: comparator (type).

- Outcomes: as specified in Types of outcome measures.

\section{Living systematic review considerations}

Two review authors will extract, evaluate, and integrate studies identified through the weekly searches.

\section{Assessment of risk of bias in included studies}

We will use the risk of bias 2 (RoB 2) tool to analyse the risk of bias in the underlying study results (Sterne 2019). Of interest for this review is the effect of the assignment to the intervention (the intentionto-treat (ITT) effect), and we will perform all assessments with RoB 2 to this effect. The outcomes that we will address are those specified for inclusion in 'Summary of findings and assessment of the certainty of the evidence'.

Two review authors (from among SJV, KLC, VP, CK, Cl and NS) will independently assess the risk of bias for each study result. In case of discrepancies between their judgements or inability to reach consensus, we will consult a third review author to reach a final decision. We will assess the following types of bias as outlined in Chapter 8 of the Cochrane Handbook (Higgins 2021c).

- Bias arising from the randomisation process.

- Bias due to deviations from the intended interventions.

- Bias due to missing outcome data.

- Bias in measurement of the outcome.

- Bias in selection of the reported result.

For cluster-RCTs, we plan to add an additional domain to assess bias arising from the timing of identification and recruitment of participants in relation to timing of randomisation, as recommended in the archived RoB 2 guidance for clusterrandomised trials (Eldridge 2016), and in Chapter 23 of the Cochrane Handbook for Systematic Reviews of Interventions (Higgins 2021b).
To address these types of bias, we will use the signalling questions recommended in RoB 2 and make a judgement using the following options.

- 'Yes': if there is firm evidence that the question is fulfilled in the study (i.e. the study is at low or high risk of bias given the direction of the question).

- 'Probably yes': a judgement has been made that the question is fulfilled in the study (i.e. the study is at low or high risk of bias given the direction of the question).

- 'No': if there is firm evidence that the question is unfulfilled in the study (i.e. the study is at low or high risk of bias for the given the direction of the question).

- 'Probably no': a judgement has been made that the question is unfulfilled in the study (i.e. the study is at low or high risk of bias given the direction of the question).

- 'No information': if the study report does not provide sufficient information to allow any judgement.

We will use the algorithms proposed by RoB 2 to assign each domain one of these levels of bias:

- low risk of bias;

- some concerns;

- high risk of bias

Subsequently, we will derive a risk of bias rating for each prespecified outcome in each study in accordance with the following suggestions.

- 'Low risk of bias': we judged the trial to be at low risk of bias for all domains for this result.

- 'Some concerns': we judged the trial to raise some concerns in at least one domain for this result, but not to be at high risk of bias for any domain.

- 'High risk of bias': we judge the trial to be at high risk of bias in at least one domain for the result or we judge the trial to have some concerns for multiple domains in a way that substantially lowers confidence in the results.

We will use the RoB 2 Excel tool to implement RoB 2 (available on the riskofbiasinfo.org website); we will add our judgements to the analysis for each assessed study and outcome; and we will store our detailed RoB 2 assessments as supplementary online material. We will use the overall risk of bias judgement, derived from the RoB 2 Excel tool, to inform our GRADE decision on downgrading for risk of bias.

\section{Measures of treatment effect}

For continuous outcomes, we will record the mean, standard deviation, and total number of participants in both the treatment and control groups. For dichotomous outcomes, we will record the number of events and total number of participants in both the treatment and control groups.

For continuous outcomes using the same scale, we will perform analyses using the mean difference (MD) with $95 \%$ confidence intervals (Cls). For continuous outcomes measured with different scales we will perform analyses using the standardised mean difference (SMD). For interpreting SMDs, we will re-express SMDs in 
the original units of a particular scale with the most clinical relevance and impact.

If available, we will extract and report hazard ratios (HRs) for timeto-event outcomes (e.g. discharge from hospital). If HRs are not available, we will make every effort to estimate the HR as accurately as possible using the available data and a purpose-built method based on the Parmar and Tierney approach (Parmar 1998; Tierney 2007). If sufficient studies provided HRs, we will use HRs rather than risk ratios (RRs) or MDs in a meta-analysis.

For dichotomous outcomes, we plan to report the pooled RR and risk difference (RD) with the associated 95\% Cls (Deeks 2019). If the number of observed events is small (less than $5 \%$ of sample per group), and if studies have balanced treatment groups, we plan to report the Peto odds ratio (OR) with 95\% Cl (Deeks 2019).

\section{Unit of analysis issues}

As recommended in Chapter 6 of the Cochrane Handbook (Higgins 2021d), for studies with multiple treatment groups, we plan to combine arms if they can be regarded as subtypes of the same intervention.

When arms cannot be pooled this way, we plan to compare each arm with the common comparator separately. For pair-wise metaanalysis, we plan to split the 'shared' group into two or more groups with smaller sample sizes, and include two or more (reasonably independent) comparisons. For this purpose, for dichotomous outcomes, both the number of events and the total number of participants will be divided up, and for continuous outcomes, the total number of participants will be divided up with unchanged means and standard deviations (SDs).

\section{Dealing with missing data}

When we identify missing data at the study level, we will contact principal investigators and request these data. If, after this, data are still missing, we will consult with content experts to judge whether data are missing at random (e.g. if missing outcomes were balanced across study arms, reasons for lost to follow-up are common and reasonable) or not. If we judge data to be missing at random, we will perform a complete case analysis and exclude the participants with missing outcome data from the analysis (Guyatt 2017). When we judge data to be not missing at random, and we identify no supporting evidence that the results were not biased by missing outcome data, we will not make any assumptions about the missing outcome data. We will conduct sensitivity analyses to assess the impact of missing data on the overall effect. We will discuss the potential impact of its absence on the results.

\section{Assessment of heterogeneity}

We will assess heterogeneity of treatment effects between trials using a $\mathrm{Chi}^{2}$ test with a significance level at $\mathrm{P}$ less than 0.1 , and visual examination. We will use the 12 statistic (Higgins 2003), to quantify possible heterogeneity ( $12>30 \%$ to signify moderate heterogeneity, $12>75 \%$ to signify considerable heterogeneity; Deeks 2019). If heterogeneity is above $80 \%$, we will explore potential causes through subgroup analyses. If we find a reason for heterogeneity, we will not perform a meta-analysis, but we will comment on results from all studies and present these in tables.

\section{Assessment of reporting biases}

As mentioned above, we will search trials registries to identify completed studies that have not been published elsewhere, to minimise or determine publication bias. We will include studies irrespective of their publication status, as recommended in Chapter 3 of the Cochrane Handbook (McKenzie 2021).

We will explore potential publication bias by generating a funnel plot and statistically testing this by conducting a linear regression test for meta-analyses involving at least 10 studies (Page 2021). We will consider a $\mathrm{P}$ value of less than 0.1 as significant for this test.

\section{Data synthesis}

If the clinical and methodological characteristics of individual studies are sufficiently homogeneous, we will pool the data in meta-analysis. We will perform separate analyses for populations with ambulatory mild disease and for hospitalised participants with moderate to severe disease, according to the latest WHO clinical progression score (WHO 2020e). We will perform analyses according to the recommendations in Chapter 10 of the Cochrane Handbook (Deeks 2019). We will not conduct meta-analyses that include different study designs. We will conduct separate meta-analyses for each comparison.

We will use the RevMan Web 2020 software for analyses (RevMan Web 2020). One review author will enter the data into the software, and a second review author will check the data for accuracy.

We will use the random-effects model for all analyses, as we anticipate that for included studies, true effects would be related, but would not be the same. For binary outcomes, we will base the estimation of the between-study variance using the MantelHaenszel method. We will use the inverse variance method for continuous outcomes, outcomes that include data from clusterRCTs, or outcomes where HRs are available. We plan to explore heterogeneity above $80 \%$ with subgroup analyses. If we cannot find a cause for the heterogeneity or if study outcomes are too clinically heterogeneous to be combined, we will not perform a meta-analysis, but comment on the results in a narrative analysis, with the results from all studies presented in tables.

\section{Living systematic review considerations}

Whenever new evidence (studies, data, or information) that meets the review inclusion criteria is identified, we will immediately assess the risk of bias and extract the data and incorporate it in the synthesis, as appropriate. We will not adjust the meta-analyses to account for multiple testing, given the methods related to frequent updating of meta-analyses are under development (Simmonds 2017).

\section{Subgroup analysis and investigation of heterogeneity}

We will perform subgroup analyses of the following characteristics for the outcome of mortality.

- Severity of condition (divided into moderate and severe disease; divided into participants receiving invasive mechanical ventilation at baseline or not).

- Duration since symptom onset (divided into up to 7 days and more than 7 days).

- Antibodies in recipients detected at baseline; that is, seropositive or seronegative (divided into detected in a 
maximum of $20 \%$ of recipients versus detected in at least $80 \%$ of recipients).

- Age of participants (divided into applicable age groups; e.g. children; 18 to 65 years, 65 years and older).

- Pre-existing conditions (diabetes, respiratory disease, hypertension, immunosuppression).

- SARS-CoV-2 variants (e.g. B1.1.7, B.1.351, P.1, and other variants that may occur in the future).

- Concentration of neutralising antibodies in the therapy (i.e. with known concentration of neutralising antibodies by taking into account batch-dependent neutralising antibody levels, or with unknown concentration).

We will use the tests for interaction to test for differences between subgroup results.

\section{Sensitivity analysis}

We will perform sensitivity analyses for the following.

- 'Risk of bias' assessment components (studies with a low risk of bias or some concerns versus studies with a high risk of bias).

- Impact of completed, but not published, studies.

- Impact of premature termination of studies.

- Impact of studies that include individuals with suspected COVID-19.

- Fixed-effect model meta-analysis.

- Impact of missing outcome data.

\section{Summary of findings and assessment of the certainty of the evidence}

We will use the GRADE approach to assess the certainty of the evidence for the following outcomes, and we will prepare one summary of findings table per population.

\section{Individuals with a confirmed diagnosis of COVID-19 and moderate to severe disease}

- All-cause mortality; all-cause mortality at hospital discharge most favourable. If not reported, all-cause mortality day 60 followed by day 28 , or time-to-event estimate, will be included in the summary of findings table.

- Worsening of clinical status at day 28:

* Participants with clinical deterioration (new need for invasive mechanical ventilation) or death.

- Improvement of clinical status at day 28:

* Participants discharged alive. Participants should be discharged without clinical deterioration or death.

- Quality of life, including fatigue and functional independence; assessed with standardised scales (e.g. WHOQOL-100) at longest follow-up available

- Any adverse events during the study period

- Serious adverse events during the study period

\section{Individuals with a confirmed diagnosis of SARS-CoV-2 infection and asymptomatic or mild disease}

- All-cause mortality; all-cause mortality at longest follow-up and greater than 60 days most favourable. If not reported, allcause mortality day 60 , followed by day 28 , or time-to-event estimate, will be included in the summary of findings table.
- Admission to hospital or death within 28 days

- Symptom resolution:

* All initial symptoms resolved (asymptomatic) at day 14;

* Duration to symptom resolution. Quality of life, including fatigue and functional independence; assessed with standardised scales (e.g. WHOQOL-100) at longest follow-up available

- Any adverse events during the study period

- Serious adverse events during the study period

We will follow the current GRADE guidance in its entirety for these assessments, as recommended in Chapter 14 of the Cochrane Handbook (Schünemann 2021). We will use GRADEpro GDT software to create a summary of findings table (Schünemann 2021). For RCTs, we will use the overall risk of bias judgement, derived from the RoB 2 Excel tool, to inform our decision on downgrading for risk of bias. We will assess the certainty of the evidence for non-controlled non-randomised studies of interventions (NRSIs) as reported in the GRADE guidance 3, starting from low-certainty evidence (Balshem 2011). For time-to-event outcomes, we will calculate absolute effects at specific time points, as recommended in the GRADE guidance 27 (Skoetz 2020). We will phrase the findings and certainty of the evidence as suggested in the informative statement guidance (Santesso 2020). For binary data, we will report relative and absolute risk differences.

\section{Methods for future updates - living systematic review considerations}

We will update our searches to monitor newly published results of RCTs on hyperimmune immunoglobulins on a weekly basis. Two review authors will screen, extract, evaluate, and integrate information following the guidance for Cochrane living systematic reviews (Brooker 2019).

We will manually check platform trials that were previously identified and listed as 'studies awaiting classification' for additional treatment arms.

We will wait until the accumulating evidence changes one or more of the following components of the review before republishing the review.

- The findings of one or more of the critical outcomes.

- The credibility (e.g. GRADE rating) of one or more of the critical outcomes.

- New settings, populations, interventions, comparisons, or outcomes studied.

\section{When review methods will be reviewed}

We will check the review scope and methods approximately monthly, or more frequently if appropriate, in light of potential changes in COVID-19 research (for example, when additional comparisons, interventions, subgroups or outcomes, or new review methods, become available).

The conditions under which the review will no longer be maintained as a living systematic review (LSR)

In our regular review of the scope, we will decide whether to continue or stop updating the review. Decisions to stop may be that the conclusions for our main outcomes and populations of interest are unlikely to change with future studies included in the review, 
no new evidence is expected, or the review question is no longer a priority for policy and practice.

\section{ACKNOWLEDGEMENTS}

This work is part of a series of reviews investigating treatments and therapies for COVID-19 as part of the project CEOsys (covidevidenz.de/what-is-ceosys/). Passages in the background section (e.g. "Description of the condition" and "Why it is important to do this review") are shared between reviews in this series. Specifically, this protocol was previously part of a parent review addressing convalescent plasma and hyperimmune immunoglobulins (hIVIG) for people with COVID-19 (Piechotta 2021). The review has been split to address hIVIG and convalescent plasma separately. Therefore, parts of the 'Background' text are shared between the two reviews. We thank the review authors of the first published reviews in this series for providing and sharing this information.

This protocol was published in collaboration with the Cochrane Editorial \& Methods Department. We particularly thank the managing editor Lara Kahale of the Central Editorial Service for her support. She managed the editorial process and contributed to the improvement of the protocol.

We thank Robin Featherstone (Information Specialist, Cochrane Editorial \& Methods Department) for commenting on the search strategy.
We thank the clinical reviewers Jeannette Guarner (Professor of Pathology and Laboratory Medicine Vice Chair of Faculty Affairs, Emory University, Department I of Internal Medicine, Division of Infectious Diseases, University of Cologne) and Michael James Ankcorn (Consultant in Virology/Infectious Diseases, Sheffield Teaching Hospitals, NHS Foundation Trust, UK) for their valuable input and critical comments. We thank Faith Armitage for providing copy-editing.

We would like to thank the consumer reviewer, Ceri Dare, in particular for her comments, which have led to better comprehensibility and readability of the review.

The research was supported by NHS Blood and Transplant and the National Institute for Health Research (NIHR) Oxford Biomedical Research Centre (BRC). The views expressed are those of the author(s) and not necessarily those of the NHS, the NIHR, or the Department of Health.

This project has received funding from the European Union's Horizon 2020 research and innovation program under grant agreement No. 101015756. The contents of this document reflect only the authors' view and the Commission is not responsible for any use that may be made of the information it contains. 


\section{RE F E R E N C E S}

\section{Additional references}

\section{Balshem 2011}

Balshem H, Helfand M, Schünemann HJ, Oxman AD, Kunz R, Brozek J, et al. GRADE guidelines: 3. Rating the quality of evidence. Journal of Clinical Epidemiology 2011;64(4):401-6.

\section{Bao 2020}

Bao L, Deng W, Gao H, Xiao C, Liu J, Xue J, et al. Reinfection could not occur in SARS-CoV-2 infected rhesus macaques. bioRxiv [Preprint] 2020 March 14. [DOI: 10.1101/2020.03.13.990226]

\section{Baudel 2020}

Baudel JL, Vigneron C, Pras-Landre V, Joffre J, Marjot F, AitOufella $\mathrm{H}$, et al. Transfusion-related acute lung injury (TRALI) after intravenous immunoglobulins: French multicentre study and literature review. Clinical Rheumatology 2020;39(2):541-6.

\section{Beigel 2020}

Beigel JH, Tomashek KM, Dodd LE, Mehta AK, Zingman BS, Kalil AC, et al. Remdesivir for the treatment of COVID-19 - preliminary report. New England Journal of Medicine 2020;383(10):992-4. [DOI: 10.1056/NEJMoa2007764]

\section{Bloch 2020}

Bloch EM, Shoham S, Casadevall A, Sachais BS, Shaz B, Winters JL, et al. Deployment of convalescent plasma for the prevention and treatment of COVID-19. Journal of Clinical Investigation 2020;130(6):2757-65.

\section{Bloch 2021}

Bloch EM. COVID-19: convalescent plasma and hyperimmune globulin. www.uptodate.com/contents/covid-19-convalescentplasma-and-hyperimmune-globulin (accessed 5 July 2021).

\section{Bonaros 2008}

Bonaros N, Mayer B, Schachner T, Laufer G, Kocher A. CMVhyperimmune globulin for preventing cytomegalovirus infection and disease in solid organ transplant recipients: a meta-analysis. Clinical Transplantation 2007;22(1):89-97. [DOI: 10.1111/j.1399-0012.2007.00750.x]

\section{Brennan 2003}

Brennan VM, Salomé-Bentley NJ, Chapel HM, Immunology Nurses Study. Prospective audit of adverse reactions occurring in 459 primary antibody-deficient patients receiving intravenous immunoglobulin. Clinical and Experimental Immunology 2003;133(2):247-51.

\section{Brooker 2019}

Brooker J, Synnot A, McDonald S, Elliott J, Turner T, Hodder R et al, . Guidance for the production and publication of Cochrane living systematic reviews: Cochrane Reviews in living mode. https://community.cochrane.org/sites/default/files/uploads/ inline-files/Transform/201912_LSR_Revised_Guidance.pdf (accessed 13 September 2021);Version December 2019.

\section{Casadevall 2020}

Casadevall A, Pirofski L. The convalescent sera option for containing COVID-19. Journal of Clinical Investigation 2020;130(4):1545-8

\section{CDC 2020}

Centers for Disease Control and Prevention (CDC). Interim clinical guidance for management of patients with confirmed coronavirus disease (COVID-19). www.cdc.gov/ coronavirus/2019-ncov/hcp/clinical-guidance-managementpatients.html (accessed 2 July 2021).

\section{Chen 2020a}

Chen L, Xiong J, Bao L, Shi Y. Convalescent plasma as a potential therapy for COVID-19. Lancet Infectious Diseases 2020;20(4):398-400.

\section{COMET 2020}

Core outcome set developers' response to COVID-19 (2 April 2020). Available from www.comet-initiative.org/Studies/ Details/1538 (accessed 2 July 2021).

\section{Covidence [Computer program]}

Veritas Health Innovation Covidence. Version accessed 2 July 2021. Melbourne, Australia: Veritas Health Innovation, 2020. Available at covidence.org.

\section{D'Antiga 2020}

D’Antiga L. Coronaviruses and immunosuppressed patients: the facts during the third epidemic. Liver Transplantation 2020;26(6):832-4.

\section{da Costa 2021}

da Costa CB, Martins FJ, da Cunha LE, Ratcliffe NA, Cisne de Paul R, Castro HC. COVID-19 and hyperimmune sera: a feasible plan B to fight against coronavirus. International Immunopharmacology 2021;90:107220. [DOI: 10.1016/ j.intimp.2020.107220]

\section{Davey 2019}

Davey RT Jr, Fernández-Cruz E, Markowitz N, Pett S, Babiker AG, Wentworth $D$, et al. Anti-influenza hyperimmune intravenous immunoglobulin for adults with influenza A or B infection (FLUIVIG): a double-blind, randomised, placebo-controlled trial. Lancet Respiratory Medicine 2019;7(11):951-63. [DOI: 10.1016/ S2213-2600(19)30253-X]

\section{Deeks 2019}

Deeks JJ, Higgins JP, Altman DG, editor(s). Chapter 10: Analysing data and undertaking meta-analyses. In: Higgins JP, Thomas J, Chandler J, Cumpston M, Li T, Page MJ, et al, editor(s). Cochrane Handbook for Systematic Reviews of Interventions Version 6.0 (updated July 2019). Cochrane, 2019. Available from www.training.cochrane.org/handbook.

\section{Driggin 2020}

Driggin E, Madhavan MV, Bikdeli B, Chuich T, Laracy J, BiondiZoccai G, et al. Cardiovascular considerations for patients, health care workers, and health systems during the COVID-19 
pandemic. Journal of the American College of Cardiology 2020;75(18):2352-71.

\section{Eldridge 2016}

Eldridge S, Campbell M, Campbell M, Dahota A, Giraudeau B, Higgins JT, et al. Revised Cochrane risk of bias tool for randomized trials (RoB 2.0). Additional considerations for cluster-randomized trials. Available from www.riskofbias.info/ welcome/rob-2-0-tool/rob-2-for-cluster-randomized-trials (accessed 2nd July 2021).

\section{Farrugia 2020}

Farrugia A, MacPherson J, Busch MP. Convalescent plasma - this is no time for competition. Transfusion 2020;60(7):1644-6.

\section{Fung 2020}

Fung M, Babik JM. COVID-19 in immunocompromised hosts: what we know so far. Clinical Infectious Diseases 2020;72:340-350. [DOI: 10.1093/cid/ciaa863]

\section{Ghosn 2021}

Ghosn L, Chaimani A, Evrenoglou T, Davidson M, Graña C, Schmucker $\mathrm{C}$, et al. Interleukin-6 blocking agents for treating COVID-19: a living systematic review. Cochrane Database of Systematic Reviews 2021, Issue 3. Art. No: CD013881. [DOI: 10.1002/14651858.CD013881]

\section{GRADEpro GDT [Computer program]}

McMaster University (developed by Evidence Prime) GRADEpro GDT. Version accessed 2 July 2021. Hamilton (ON): McMaster University (developed by Evidence Prime). Available at gradepro.org.

\section{Guyatt 2017}

Guyatt GH, Ebrahim S, Alonso-Coello P, Johnston BC, Mathioudakis AG, Briel M, et al. GRADE guidelines 17: assessing the risk of bias associated with missing participant outcome data in a body of evidence. Journal of Clinical Epidemiology 2017;87:14-22. [DOI: https://doi.org/10.1016/ j.jclinepi.2017.05.005]

\section{Higgins 2003}

Higgins JP, Thompson SG, Deeks JJ, Altman DG. Measuring inconsistency in meta-analyses. BMJ 2003;327:557-60.

\section{Higgins 2021a}

Higgins JP, Thomas J, Chandler J, Cumpston M, Li T, Page MJ, et al, editor(s). Cochrane Handbook for Systematic Reviews of Interventions Version 6.2 (updated February 2021). Cochrane, 2021. Available from www.training.cochrane.org/handbook.

\section{Higgins 2021b}

Higgins JP, Eldridge S, Li T (editors). Chapter 23: Including variants on randomized trials. In: Higgins JP, Thomas J, Chandler J, Cumpston M, Li T, Page MJ, et al (editors). Cochrane Handbook for Systematic Reviews of Interventions version 6.2 (updated February 2021). Cochrane, 2021. Available from www.training.cochrane.org/handbook.

\section{Higgins 2021c}

Higgins JP, Savović J, Page M, Elbers RG, Sterne JA. Chapter 8: Assessing risk of bias in a randomized trial. In: Higgins JP, Thomas J, Chandler J, Cumpston M, Li T, Page MJ, et al (editors). Cochrane Handbook for Systematic Reviews of Interventions version 6.2 (updated February 2021). Cochrane, 2021. Available from www.training.cochrane.org/handbook.

\section{Higgins 2021d}

Higgins JP, Deeks JJ, editor(s). Chapter 6: Choosing effect measures and computing estimates of effect. In: Higgins JP, Thomas J, Chandler J, Cumpston M, Li T, Page MJ, et al, editor(s). Cochrane Handbook for Systematic Reviews of Interventions Version 6.2 (updated February 2021). Cochrane, 2021. Available from www.training.cochrane.org/handbook.

\section{Ho 2005}

Ho MS, Chen WJ, Chen HY, Lin SF, Wang MC, Di J, et al. Neutralizing antibody response and SARS severity. Emerging Infectious Diseases 2005;11(11):1730-7.

\section{Horby 2020}

Horby P, Lim WS, Emberson J, Mafham M, Bell J, Linsell L, et al. Effect of dexamethasone in hospitalized patients with COVID-19: preliminary report. medRxiv [Preprint] 2020 June 22. [DOI: doi.org/10.1101/2020.06.22.20137273]

\section{Horby 2021}

Horby PW, Mafham M, Peto L, Campbell M, Pessoa-Amorim G, et al, RECOVERY Collaborative Group. Casirivimab and imdevimab in patients admitted to hospital with COVID-19 (RECOVERY): a randomised, controlled, open-label, platform trial. medRxiv [Preprint] 2021 June 16. [DOI: https:// doi.org/10.1101/2021.06.15.21258542]

\section{Huang 2020}

Huang C, Wang Y, Li X, Ren L, Zhao J, Hu Y, et al. Clinical features of patients infected with 2019 novel coronavirus in Wuhan, China. Lancet 2020;395(10223):497-506. [DOI: 10.1016/ S0140-6736(20)30183-5]

\section{Hung 2013}

Hung IF, To KK, Lee CK, Lee KL, Yan WW, Chan K, et al. Hyperimmune IV immunoglobulin treatment: a multicenter double-blind randomized controlled trial for patients with severe 2009 influenza $A(H 1 N 1)$ infection. Chest 2013;144(2):464-73. [DOI: 10.1378/chest.12-2907]

\section{Iwasaki 2021}

Iwasaki A. What reinfections mean for COVID-19. Lancet Infectious Diseases 2021;21(1):3-5.

\section{Izquierdo-Dominguez 2020}

Izquierdo-Dominguez A, Rojas-Lechuga MJ, Mullol J, Alobid I. Olfactory dysfunction in the COVID-19 outbreak. Journal of Investigational Allergology \& Clinical Immunology 2020;30(5):317-326. [DOI: 10.18176/jiaci.0567] 


\section{Johns Hopkins 2021}

Johns Hopkins University and Medicine. Mortality analyses. Available from coronavirus.jhu.edu/data/mortality (accessed 2 July 2021).

\section{Joyner 2021}

Joyner MJ, Carter RE, Senefeld JW, Klassen SA, Mills JR, Johnson PW, et al. Convalescent plasma antibody levels and the risk of death from Covid-19. New England Journal of Medicine 2021;384:1015-27. [DOI: 10.1056/NEJMoa2031893]

\section{Kim 2020}

Kim D-H, Choe YJ, Jeong J-Y. Understanding and interpretation of case fatality rate of coronavirus disease 2019. Journal of Korean Medical Science 2020;35(12):e137. [DOI: 10.3346/ jkms.2020.35.e137]

\section{Kreuzberger 2021}

Kreuzberger N, Hirsch C, Chai KL, Piechotta V, Valk SJ, Estcourt LJ, et al. SARS-CoV-2-neutralising monoclonal antibodies for treatment of COVID-19. Cochrane Database of Systematic Reviews 2021, Issue 1. Art. No: CD013825. [DOI: 10.1002/14651858.CD013825]

\section{Lauer 2020}

Lauer SA, Grantz KH, Bi Q, Jones FK, Zheng Q, Meredith HR, et al. The incubation period of coronavirus disease 2019 (COVID-19) from publicly reported confirmed cases: estimation and application. Annals of Internal Medicine 2020;172(9):577-582. [DOI: 10.7326/M20-0504]

\section{Lefebvre 2021}

Lefebvre C, Glanville J, Briscoe S, Littlewood A, Marshall C, Metzendorf MI, et al. Chapter 4: Searching for and selecting studies. In: Higgins JP, Thomas J, Chandler J, Cumpston M, Li T, Page MJ, et al, editor(s). Cochrane Handbook for Systematic Reviews of Interventions Version 6.2 (updated February 2021). Cochrane, 2021. Available from www.training.cochrane.org/ handbook.

\section{Li 2021}

Li T, Higgins JP, Deeks JJ, editor(s). Chapter 5: Collecting data. In: Higgins JP, Thomas J, Chandler J, Cumpston M, Li T, Page MJ, et al, editor(s). Cochrane Handbook for Systematic Reviews of Interventions Version 6.2 (updated February 2021). Cochrane, 2021. Available from www.training.cochrane.org/handbook.

\section{Liang 2020}

Liang W, Guan W, Chen R, Wang W, Li J, Xu K, et al. Cancer patients in SARS-CoV-2 infection: a nationwide analysis in China. Lancet Oncology 2020;21(3):335-7. [DOI: 10.1016/ S1470-2045(20)30096-6]

\section{Maatman 2020}

Maatman TK, Jalali F, Feizpour C, Douglas A 2nd. Routine venous thromboembolism prophylaxis may be inadequate in the hypercoagulable state of severe coronavirus disease 2019. Critical Care Medicine 2020;48(9):e783-e790. [DOI: 10.1097/ CCM.0000000000004466]

\section{McKenzie 2021}

McKenzie JE, Brennan SE, Ryan RE, Thomson HJ, Johnston RV, Thomas J. Chapter 3: Defining the criteria for including studies and how they will be grouped for the synthesis. In: Higgins JP, Thomas J, Chandler J, Cumpston M, Li T, Page MJ, et al (editors). Cochrane Handbook for Systematic Reviews of Interventions version 6.2 (updated February 2021). Cochrane, 2021. Available from www.training.cochrane.org/handbook.

\section{Microsoft Corporation 2018 [Computer program]}

Microsoft Excel. Microsoft Corporation, 2018. Available at office.microsoft.com/excel.

\section{Mo 2006}

Mo H, Zeng G, Ren X, Li H, Ke C, Tan Y, et al. Longitudinal profile of antibodies against SARS-coronavirus in SARS patients and their clinical significance. Respirology 2006;11(1):49-53. [DOI: 10.1111/j.1440-1843.2006.00783.x]

\section{Moher 2009}

Moher D, Liberati A, Tetzlaff J, Altman DG. Preferred reporting items for systematic reviews and meta-analyses: the PRISMA statement. Journal of Clinical Epidemiology 2009;62(10):1006-12.

\section{Morens 1994}

Morens DM. Antibody-dependent enhancement of infection and the pathogenesis of viral disease. Clinical Infectious Diseases 1994;19(3):500-12.

\section{NCPERE 2020}

NCPERE (Novel Coronavirus Pneumonia Emergency Response Epidemiology) team. Vital surveillances: the epidemiological characteristics of an outbreak of 2019 novel coronavirus diseases (COVID-19) - China. China CDC Weekly 2020;2(8):113-22. [DOI: 10.3760/ cma.j.issn.0254-6450.2020.02.003]

\section{Page 2021}

Page MJ, Higgins JP, Sterne JA. Chapter 13: Assessing risk of bias due to missing results in a synthesis. In: Higgins JP, Thomas J, Chandler J, Cumpston M, Li T, Page MJ, et al (editors). Cochrane Handbook for Systematic Reviews of Interventions version 6.2 (updated February 2021). Cochrane, 2021. Available from www.training.cochrane.org/handbook.

\section{Parmar 1998}

Parmar MK, Torri V, Stewart L. Extracting summary statistics to perform meta-analyses of the published literature for survival endpoints. Statistics in Medicine 1998;17(24):2815-34.

\section{Payne 2016}

Payne DC, Iblan I, Rha B, Algasrawi S, Hin A, Al Nsour M, et al. Persistence of antibodies against Middle East respiratory syndrome coronavirus. Emerging Infectious Disease Journal 2016;22(10):1824-6. [DOI: 10.3201/eid2210.160706]

\section{PHE 2019}

Public Health England. Updated guidelines on post exposure prophylaxis (PEP) for varicella/shingles; June 2019. Available at assets.publishing.service.gov.uk/government/uploads/ 
system/uploads/attachment_data/file/812526/PHE_PEP_ VZIG_guidance_for_health_professionals.pdf.

\section{Piechotta 2021}

Piechotta V, Iannizzi C, Chai KL, Valk SJ, Kimber C, Dorando E, et al. Convalescent plasma or hyperimmune immunoglobulin for people with COVID-19: a living systematic review. Cochrane Database of Systematic Reviews 2021, Issue 5. Art. No: CD013600. [DOI: 10.1002/14651858.CD013600.pub4]

\section{RevMan Web 2020 [Computer program]}

The Cochrane Collaboration Review Manager Web (RevMan Web). Version 1.22.0. The Cochrane Collaboration, 2020. Available at revman.cochrane.org.

\section{Robbins 1995}

Robbins JB, Schneerson R, Szu SC. Perspective: hypothesis: serum IgG antibody is sufficient to confer protection against infectious diseases by inactivating the inoculum. Journal of Infectious Diseases 1995;171(6):1387-98.

\section{Santesso 2020}

Santesso N, Glenton C, Dahm P, Garner P, Akl A, Alper B, et al. GRADE guidelines 26: informative statements to communicate the findings of systematic reviews of interventions. Journal of Clinical Epidemiology 2020;119:126-35.

\section{Schünemann 2021}

Schünemann HJ, Higgins JP, Vist GE, Glasziou P, Akl EA, Skoetz N, et al. Chapter 14: Completing 'Summary of findings' tables and grading the certainty of the evidence. In: Higgins JP, Thomas J, Chandler J, Cumpston M, Li T, Page MJ, et al (editors). Cochrane Handbook for Systematic Reviews of Interventions version 6.2 (updated February 2021). Cochrane, 2021. Available from www.training.cochrane.org/handbook.

\section{Sekul 1994}

Sekul EA, Cupler EJ, Dalakas MC. Aseptic meningitis associated with high-dose intravenous immunoglobulin therapy: frequency and risk factors. Annals of Internal Medicine 1994;121(4):259-62.

\section{Sharma 2021}

Sharma A, Bhatt NS, St Martin A, Abid MB, Bloomquist J, Chemaly RF, et al. Clinical characteristics and outcomes of COVID-19 in haematopoietic stem-cell transplantation recipients: an observational cohort study. Lancet Haematology 2021;8(3):e185-93.

\section{Shen 2020}

Shen C, Wang Z, Zhao F, Yang Y, Li J, Yuan J, et al. Treatment of 5 critically ill patients with COVID-19 with convalescent plasma. JAMA 2020;323(16):1582-9.

\section{Simmonds 2017}

Simmonds M, Salanti G, McKenzie J, Elliott J, Living Systematic Review Network. Living systematic reviews: 3. Statistical methods for updating meta-analyses. Journal of Clinical Epidemiology 2017;91:38-46. [DOI: 10.1016/ j.jclinepi.2017.08.008]

\section{Skoetz 2020}

Skoetz N, Goldkuhle M, Van Dalen EC, Akl EA, Trivella M, Mustafa RA, et al. GRADE guidelines 27: how to calculate absolute effects for time-to-event outcomes in summary of findings tables and evidence profiles. Journal of Clinical Epidemiology 2020;118:124-31.

\section{Sterne 2019}

Sterne JA, Savovic J, Page MJ, Elbers RG, Blencowe NS, Boutron I, et al. RoB 2: a revised tool for assessing risk of bias in randomised trials. BMJ 2019;366:14898.

\section{Stiehm 2013}

Stiehm ER. Adverse effects of human immunoglobulin therapy. Transfusion Medicine Reviews 2013;27(3):171-8.

\section{Tierney 2007}

Tierney JF, Stewart LA, Ghersi D, Burdett S, Sydes MR. Practical methods for incorporating summary time-to-event data into meta-analysis. Trials 2007;8:16.

\section{Tolouian 2020}

Tolouian R, Vahed SZ, Ghiyasvand S, Tolouian A, Ardalan M. COVID-19 interactions with angiotensin-converting enzyme 2 (ACE2) and the kinin system; looking at a potential treatment. Journal of Renal Injury Prevention 2020;9(2):e19. [DOI: 10.34172/ jrip.2020.19]

\section{Vandeberg 2021}

Vandeberg P, Cruz M, Diez JM, Merritt WK, Santos B, Trukawinski S, et al. Production of anti-SARS-CoV-2 hyperimmune globulin from convalescent plasma. Transfusion 2021;61(6):1705-9.

\section{Van de Veerdonk 2020}

Van de Veerdonk F, Netea MG, Van Deuren M, Van der Meer JW, De Mast Q, Bruggemann RJ, et al. Kinins and cytokines in COVID-19: a comprehensive pathophysiological approach. Preprints [Preprint] 2020 April 3. [DOI: 10.20944/ preprints202004.0023.v1]

\section{Wan 2020}

Wan Y, Shang J, Sun S, Tai W, Chen J, Geng Q, et al. Molecular mechanism for antibody-dependent enhancement of coronavirus entry. Journal of Virology 2020;94(5):e02015-9.

\section{Wang 2014}

Wang S-F, Tseng S-P, Yen C-H, Yang J-Y, Tsao C-H, Shen C-W, et al. Antibody-dependent SARS coronavirus infection is mediated by antibodies against spike proteins. Biochemical and Biophysical Research Communications 2014;451(2):208-14.

\section{WHO 2007}

World Health Organization (WHO). Cumulative number of reported probable cases of SARS. Available from www.who.int/ csr/sars/country/2003_07_11/en/ (accessed 22 March 2021).

\section{WHO 2019}

World Health Organization (WHO). Middle East respiratory syndrome coronavirus (MERS-CoV). Available from 
www.who.int/emergencies/mers-cov/en/ (accessed 2 July 2021).

\section{WHO 2020a}

World Health Organization (WHO). Report of the WHO-China Joint Mission on coronavirus disease 2019 (COVID-19); February 2020. Available from www.who.int/docs/default-source/ coronaviruse/who-china-joint-mission-on-covid-19-final-report (accessed 2 July 2021).

\section{WHO 2020b}

World Health Organization (WHO). Rolling updates on coronavirus diseases (COVID-19). Available from www.who.int/ emergencies/diseases/novel-coronavirus-2019/events-as-theyhappen (accessed 2 July 2021).

\section{WHO 2020c}

World Health Organization (WHO). Coronavirus disease (COVID-19) situation report-209; 16 August 2020. Available from www.who.int/docs/default-source/coronaviruse/situationreports/20200816-covid-19-sitrep-209.pdf?sfvrsn=5dde1ca2_2 (accessed 2 July 2021).

\section{WHO 2020d}

World Health Organization (WHO). Clinical management of severe acute respiratory infection (SARI) when COVID19 disease is suspected: interim guidance; March 2020. Available from apps.who.int/iris/handle/10665/331446 (accessed 2 July 2021).

\section{WHO 2020e}

WHO Working Group on the Clinical Characterisation and Management of COVID-19 infection. A minimal common outcome measure set for COVID-19 clinical research. Lancet Infectious Diseases 2020;20(8):e192-7.

\section{WHO 2020 f}

World Health Organization (WHO). COVID-19 Therapeutic Trial Synopsis; February 2020. Available from www.who.int/blueprint/priority-diseases/key-action/ COVID-19_Treatment_Trial_Design_Master_Protocol_synopsis_Final_18022020.pdf (accessed 2 July 2021).

\section{ADDITIONAL TABLES}

\section{Table 1. WHO clinical progression scale}

\begin{tabular}{lll}
\hline Patient State & Descriptor & Score \\
\hline Uninfected & Uninfected; no viral RNA detected & 0 \\
\hline $\begin{array}{l}\text { Ambulatory mild dis- } \\
\text { ease }\end{array}$ & Asymptomatic; viral RNA detected & 1 \\
\cline { 2 - 3 } & Symptomatic; independent & 2 \\
\cline { 2 - 3 } & Symptomatic; assistance needed & 3 \\
\hline Hospitalised: moderate & Hospitalised; no oxygen therapy $a$ & 4 \\
\cline { 2 - 3 } & Hospitalised; oxygen by mask or nasal prongs & 5 \\
\hline
\end{tabular}

\section{Wiersinga 2020}

Wiersinga WJ, Rhodes A, Cheng AC, Peacock SJ, Prescott HC. Pathophysiology, transmission, diagnosis, and treatment of coronavirus disease 2019 (COVID-19): a review. JAMA 2020;324(8):782-93.

\section{Williamson 2020}

Williamson E, Walker AJ, Bhaskaran KJ, Bacon S, Bates C, Morton CE, et al, the OpenSAFELY Collaborative. OpenSAFELY: factors associated with COVID-19-related hospital death in the linked electronic health records of 17 million adult NHS patients. medRxiv [Preprint] 2020 May 07. [DOI: doi.org/10.1101/2020.05.06.20092999]

\section{Wu 2020a}

Wu C, Chen X, Cai Y, Xia J, Zhou X, Xu S, et al. Risk factors in patients with coronavirus disease 2019 pneumonia in Wuhan, China. JAMA Internal Medicine 2020;180:934-943. [DOI: 10.1001/ jamainternmed.2020.0994]

\section{Wu 2020b}

Wu F, Wang A, Liu M, Wang Q, Chen J, Xia S, et al. Neutralizing antibody responses to SARS-CoV-2 in a COVID-19 recovered patient cohort and their implications. medRxiv [Preprint] 2020 April 20. [DOI: 110.1101/2020.03.30.2004736]

\section{Xu 2020a}

Xu Z, Shi L, Wang Y, Zhang J, Huang L, Zhang C, et al. Pathological findings of COVID-19 associated with acute respiratory distress syndrome. Lancet Respiratory Medicine 2020;8(4):420-2. [DOI: 10.1016/S2213-2600(20)30076-X]

\section{Zhao 2020a}

Zhao J, Yuan Q, Wang H, Liu W, Liao X, Su Y, et al. Antibody responses to SARS-CoV-2 in patients of novel coronavirus disease 2019. medRxiv [Preprint] 2020 March 03. [DOI: 10.1101/2020.03.02.20030189] associated with acute respiratory distress syndrome and death 


\section{Table 1. WHO clinical progression scale (Continued)}

Hospitalised: severe disease
Hospitalised; oxygen by non-invasive mechanical ventilation or high flow

\begin{tabular}{ll}
\hline Intubation and mechanical ventilation; $\mathrm{pO}_{2} / \mathrm{FiO}_{2} \geq 150$ or $\mathrm{SpO}_{2} / \mathrm{FiO}_{2} \geq 200$ & 7 \\
\hline $\begin{array}{l}\text { Invasive mechanical ventilation; } \mathrm{pO}_{2} / \mathrm{FiO}_{2}<150\left(\mathrm{SpO}_{2} / \mathrm{FiO}_{2}<200\right) \text { or vaso- } \\
\text { pressors }\end{array}$ & 8
\end{tabular}

Invasive mechanical ventilation; $\mathrm{pO}_{2} / \mathrm{FiO}_{2}<150$ and vasopressors, dialysis or 9 ECMO

World Health Organization (WHO) clinical progression scale from: WHO 2020e

alf hospitalised for isolation only, record status as for ambulatory patient.

Abbreviations: ECMO: extracorporeal membrane oxygenation; $\mathrm{FiO}_{2}$ : fraction of inspired oxygen; $\mathrm{pO}_{2}$ : partial pressure of oxygen; $\mathrm{SpO}$ : oxygen saturation

\section{AP PE N DICES}

\section{Appendix 1. Glossary of abbreviations and medical terms}

\section{Abbreviations}

ACE2 - angiotensin-converting enzyme 2, a protein on the surface of many cells in the body

ARDS - acute respiratory distress syndrome, severe inflammation of the lungs following infection or injury

GRADE - Grading of Recommendations, Assessment, Development and Evaluation, a method of assessing the level of certainty about clinical evidence

hIVIG - hyperimmune intravenous immunoglobulin, the intervention of interest in this review

IgG - immunoglobulin G, one of the five major classes of immunoglobulins

MERS - Middle East respiratory syndrome, a coronavirus disease related to COVID-19

NRSI - non-randomised study of an intervention, a trial which compares two or more groups, but patients are not assigned to the groups randomly

pH - power of hydrogen, a standard scale for measuring acidity

PRISMA - Preferred Reporting Items for Systematic Reviews and Meta-Analyses, a standard set of items that should appear within a systematic review

REGN-COV2 - brand name of Regeneron Pharmaceuticals antibody cocktail Casirivimab/imdevimab, used to treat COVID-19

RoB 2 - Risk of bias 2, a tool for assessing the methodological quality of included studies

SARS - severe acute respiratory syndrome, a coronavirus disease related to COVID-19

SARS-CoV-2 - severe acute respiratory syndrome coronavirus 2, the virus that causes COVID-19

TACO - transfusion-associated circulatory overload, a serious transfusion reaction, defined as acute or worsening swelling in the lungs during transfusion, or up to 12 hours afterwards. Alongside this, there can be cardiovascular changes which are caused by the transfusion and not by a patient's underlying condition.

TAD - transfusion-associated dyspnoea, difficulty in breathing caused by the body reacting to transfusion

TRALI - transfusion-related acute lung injury, a serious transfusion reaction defined as a new and sudden lung injury, occurring within six hours of transfusion 
WHO - World Health Organization, the United Nations agency dedicated to health promotion

WHOQOL-100 - a 100-question standardised scale for assessing quality of life

\section{Medical terms relating to hIVIG}

Antibody-dependent enhancement - a phenomenon where antibody treatment may cause the symptoms caused by the virus to be more severe

Antibody titre - the level of concentration of antibodies in blood or plasma

Antigen-binding antibody fragments / fragment antibodies - antibodies that have been manipulated in a laboratory to make a smaller molecule

Anaphylactic reaction - a serious allergic reaction involving the IgE-producing cells in the immune system

Anaphylactoid reaction - a serious allergic reaction not involving the IgE-producing cells in the immune system

T cells - cells in the immune system produced in the thymus

Coagulation factors - proteins in the blood which control bleeding

Convalescent plasma - plasma from a person who has recovered from COVID-19, containing the antibodies they made to the disease

Erythema - skin redness caused by inflammation of small blood vessels

Granulocytic cells - a type of immune cell which secretes enzymes during infection

Haemolytic reaction - a serious reaction to transfusion, where the recipient's body destroys the donated cells

Hyperimmune animal sera - a product containing antibodies extracted and produced from animals immunised with live virus

Macrophages - a type of white blood cell which removes dead cells

Microbodies - antibodies that have been manipulated in a laboratory to be smaller and more consistent

Monoclonal antibody therapy - a type of antibody originating from identical B-cells, produced in a laboratory

Monocytes - the largest type of white blood cell

Nanobodies - antibodies that have been manipulated in a laboratory to be smaller and more consistent

Pathogen-specific neutralising antibodies - antibodies to a specific illness, which prevent virus cells from entering healthy cells in the body

Pleural effusion - build up of fluid around the lungs

Polyclonal antibodies - a mixture of several antibodies, which originate from different B-cells, and is prepared in a laboratory

Post-exposure prophylaxis - a treatment given to someone who has come into contact with COVID-19 but does not have the disease, to prevent them from becoming ill

Pre-exposure prophylaxis - a treatment given to someone who does not have COVID-19 but might come into contact with it in the future, to prevent them from becoming ill

Pulmonary embolism - a blood clot in the lungs

Pulmonary oedema - fluid in the lungs

Seronegative - the patient does not have detectable antibodies to the virus in their blood

Seropositive - the patient has detectable antibodies to the virus in their blood

Standard intravenous immunoglobulin - a product produced from donated plasma, containing antibodies

Transfusion-transmitted infection - an infection caused by bacteria, virus, or parasite in a donated blood product 


\section{Appendix 2. Search strategy MEDLINE}

\#

1

3 CoV 2" or SARSCoV2 or "SARSCoV 2").tw,kf.

4

5

7

8

9 Immunoglobulins/

10 Immunoglobulins, Intravenous/

11 Immune Sera/

12 ((convalesc ${ }^{\star}$ or recovered or cured or rehabilitat ${ }^{\star}$ or survivor* ${ }^{\star}$ or survived or virus-positive or virus neutrali* or virus inactivated or

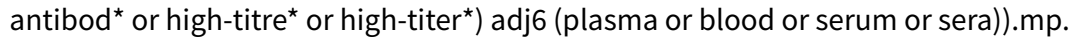

13 ((plasma adj1 therap*) or gamma-globulin* or "Y-Globulin" or hyper-lg).tw,kf.

14 (hyperimmune* or hyper-immune*).mp.

15 (high-dos* adj3 (plasma or immunoglobulin* or IVIG* or immune globulin* or globulin* or IgG)).tw,kf.

16 (plasma adj5 (immun* or antibod* or exchange* or donor* or donat* or transfus* or infus ${ }^{\star}$ )).mp.

17 ((convalesc ${ }^{\star}$ or recovered or cured or rehabilitat ${ }^{\star}$ or survivor* or survived or virus-positive or virus inactivated or antibody-positive) $\operatorname{adj5}\left(\right.$ donor $^{\star}$ or donat $\left.\left.^{\star}\right)\right) \cdot \mathrm{mp}$.

18 (serum or sera or serotherap* or sero-therap $\left.{ }^{\star}\right) . t w, k f$.

exp Immunization, Passive/

(passiv* adj3 (antibod* transfer* or immunization* or immunotherap ${ }^{\star}$ or immuno-therap* or vaccin*)).tw,kf. ((immunoglobulin* or immune globulin*) adj2 (therap* or treatment* or neutrali?ing or prevent* or protect* or prophylax*)).tw,kf. hIVIG.tw,kf.

(XAV-19 or SAB-185 or equine or INM005 or CSL760).tw,kf.

(IGY-110 or IGY110 or GIGA-2050 or GIGA2050).tw,kf.

(GC5131 or 5131A).tw,kf.

(((anti-coronavirus or anticoronavirus) adj1 immunoglobulin $\left.{ }^{\star}\right)$ or ITAC).tw,kf.

("Hyperimmune anti-COVID-19 IVIG" or C-IVIG or CIVIG).tw,kf.

(equine polyclonal antibod* or EpAbs).tw,kf.

Flebogamma.tw,kf.

or/8-29

7 and 30 
"Covid-19 Serotherapy".px.

33 (Flu-IVIG or ((anti-flu* or anti-influenza* or antiflu* or antinfluenza*) adj5 plasma)).mp.

34 studies or follow-up*).mp.

or/35-36

(animals/ not humans/) or editorial/ or exp review/ or meta analysis/ or consensus/ or exp guideline/ hi.fs. or case report.mp.

40 or/38-39

41

42

43

\section{Appendix 3. Search strategy Embase}

\section{\# Searches}

1 coronavirinae/ or coronaviridae/ or coronaviridae infection/

2 coronavirus disease 2019/

3 Coronavirus infection/

4 sars-related coronavirus/

"Severe acute respiratory syndrome coronavirus 2"/ 
$6 \quad\left(\left(\right.\right.$ corona $^{\star}$ or corono* $) \operatorname{adj1}\left(\right.$ virus $^{\star}$ or viral ${ }^{\star}$ or virinae $\left.\left.{ }^{\star}\right)\right) . t w, k w$.

7 ("2019 nCoV" or 2019nCoV or coronavir* or coronovir* or COVID or COVID19 or HCoV* or "nCov 2019" or "SARS CoV2" or "SARS CoV 2" or SARSCoV2 or "SARSCoV 2").tw,kw.

$8 \quad$ "Severe acute respiratory syndrome coronavirus 2 ".mp.

9 (anti-flu* or anti-influenza* or antiflu* or antifluenza*).tw,kw.

10 or $1-9$

11 Plasma Transfusion/

12 exp Immunoglobulin/

13 ((convalesc* or recovered or cured or survivor* or survived or rehabilitat* or virus-positive or virus-neutrali* or virus inactived or antibody-rich or high-tire* or high-titer ${ }^{\star}$ ) adj6 (plasma or blood or serum or sera)).mp.

14 ((plasma adj1 therap*) or gamma-globulin or "y-Globulin" or hyper-lg).tw,kw.

15 (plasma adj5 (immun* or antibod ${ }^{\star}$ or exchange* or donor $^{\star}$ or donat ${ }^{\star}$ or transfus $^{\star}$ or infus $\left.{ }^{\star}\right)$ ).mp.

16 (hyperimmune* or hyper-immune $\left.{ }^{\star}\right) \cdot \mathrm{mp}$.

17 (high-dos* adj3 (plasma or immunoglobulin* or IVIG* or immune globulin* or globulin* or IgG)).tw,kw.

18 ((convalesc ${ }^{\star}$ or recovered or cured or rehabilitat ${ }^{\star}$ or survivor ${ }^{\star}$ or survived or virus-positive or virus inactivated or antibody-positive) $\operatorname{adj5}\left(\right.$ donor $^{\star}$ or donat $\left.\left.^{\star}\right)\right) \cdot \mathrm{mp}$.

19 (serum or sera or serotherap* or sero-therap*).tw,kw.

20 passive immunization/

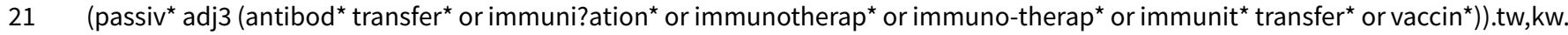

22 passive immunit* $. \mathrm{tw}, \mathrm{kw}$.

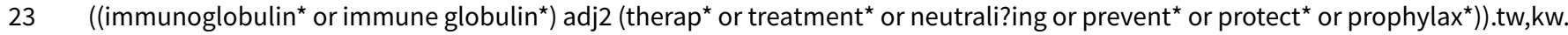

24 hIVIG.tw,kw.

(CSL760 or INM005 or XAV-19 or SAB-185 or equine).tw,kw.

(IgY-110 or IgY110 or GIGA-2050 or GIGA2050).tw,kw.

(GC5131 or 5131A).tw,kw.

(((anti-coronavirus or anticoronavirus) adj1 immunoglobulin $\left.{ }^{\star}\right)$ or ITAC).tw,kw.

("Hyperimmune anti-COVID-19 IVIG" or C-IVIG or CIVIG).tw,kw.

(equine polyclonal antibod* or EpAbs).tw,kw.

flebogamma.tw,kw.

or/11-31

(Flu-IVIG or ((anti-flu* or anti-influenza* or antiflu* or antinfluenza*) adj5 plasma)).mp.

Clinical study/

(cross sectional adj (study or studies)).tw.

exp longitudinal study/

exp prospective study/

exp follow up/ 
39

40

41

42 \$1)).ti,ab.

56 (controlled adj7 (study or design or trial)).ti,ab.

57 (volunteer or volunteers).ti,ab.

58 trial.ti

59 or/43-58

60 phase 3 clinical trial/

61 ("Phase 3" or "phase3" or "phase III" or P3 or "PIII").tw,kw.

62 or/60-61

63 (10 and 32) or 33

6463 and (42 or 59 or 62$)$

65 limit 64 to $y r=" 2019$-Current"

\section{Appendix 4. Search strategy Cochrane COVID-19 Study Register}

plasma OR convalesc* OR serum OR sera OR donor* OR donation* OR serotherapy OR "sero-therapy" OR "flu-IVIG" OR antiflu* OR "antiflu" OR hyperimmune* OR hyper-immune* OR IVIG OR immunoglobulin OR "immune-globulin" OR globulin OR "gamma-globulin" OR "Y-Globulin" OR "hyper-Ig" OR immunization OR immunisation OR immunotherap* OR CSL760 OR INM005 OR equine OR "XAV-19" OR "SAB-185" OR hIVIG OR equine OR INOSARS OR "GIGA-2050" or GIGA2050 OR "IGY-110" OR IGY1109 OR "GC5131" OR "5131A" OR ITAC OR "C-IVIG" OR CIVIG OR flebogamma OR EpAbs

Limits: treatment and management

\section{Appendix 5. Search strategy PubMed}

\#1 "2019 ncov"[Title/Abstract] OR "2019nCoV"[Title/Abstract] OR "corona virus"[Title/Abstract] OR "corona viruses"[Title/ Abstract] OR "Coronavirus"[Title/Abstract] OR "coronaviruses"[Title/Abstract] OR "COVID"[Title/Abstract] OR "COVID19"[Title/Abstract]

Hyperimmune immunoglobulin for people with COVID-19 (Protocol) 
OR "ncov 2019"[Title/Abstract] OR "SARS-CoV2"[Title/Abstract] OR "SARS-CoV-2"[Title/Abstract] OR "SARSCoV2"[Title/Abstract] OR "SARSCoV-2"[Title/Abstract] OR "COVID-19"[MeSH Terms] OR "Coronavirus"[MeSH Terms:noexp] OR "SARS-CoV-2"[MeSH Terms] OR "COVID-19"[Supplementary Concept] OR "severe acute respiratory syndrome coronavirus 2"[Supplementary Concept]

\#2 ("convalesc*"[Title/Abstract] OR "recovered"[Title/Abstract] OR "cured"[Title/Abstract] OR "rehabilitat*"[Title/Abstract] OR "survivor*"[Title/Abstract] OR "survived"[Title/Abstract] OR "virus-positive"[Title/Abstract] OR "virus neutrali*"[Title/Abstract] OR "virus inactivated"[Title/Abstract] OR "antibod*"[Title/Abstract] OR "high titre*"[Title/Abstract] OR "high titer*"[Title/Abstract]) AND ("plasma"[Title/Abstract] OR "blood"[Title/Abstract] OR "donor*"[Title/Abstract] OR "donat*"[Title/Abstract])

\#3 ("plasma"[Title] AND ("immun*"[Title/Abstract] OR "transfus*"[Title/Abstract] OR "infus*"[Title/Abstract]))

\#4 ("high dos*"[Title/Abstract] AND ("plasma"[Title/Abstract] OR "immunoglobulin*"[Title/Abstract] OR "ivig*"[Title/Abstract] OR "immune globulin*"[Title/Abstract] OR "globulin*"[Title/Abstract] OR "IgG"[Title/Abstract])

\#5 "serum"[Title] OR "sera"[Title] OR "immunization, passive"[MeSH Terms] OR "hyperimmune"[Title/Abstract] OR "hyperimmunity"[Title/Abstract] OR "serotherap*"[Title/Abstract] OR "sero therap*"[Title/Abstract] OR "therapeutic plasma"[Title/ Abstract] OR "plasma therapy"[Title/Abstract] OR "immune plasma"[Title/Abstract] OR "plasma exchange"[Title/Abstract] OR "gamma globulin*"[Title/Abstract] OR "gamma-Globulin"[Title/Abstract] OR "hyper-Ig"[Title/Abstract]

\#6 ("passiv*"[Title/Abstract] AND (("antibod*"[All Fields] AND "transfer*"[All Fields]) OR "immunisation*"[All Fields] OR "vaccin*"[Title/ Abstract] OR "immunization*"[All Fields] OR "immunotherap*"[All Fields] OR "immuno therap*"[All Fields] OR "vaccin*"[All Fields])

\#7 ("immunoglobulin*"[Title] OR "immune globulin*"[Title]) AND ("therap*"[Title/Abstract] OR "treat*"[Title/Abstract] OR "prevent*"[Title/Abstract] OR "protect*"[Title/Abstract] OR "prophylax*"[Title/Abstract]))

\#8 ("equine*"[Title/Abstract] OR "hivig*"[Title/Abstract] OR "c ivig*"[Title/Abstract] OR "XAV-19"[Title/Abstract] OR "5131A"[Title/ Abstract] OR "equine polyclonal antibod*"[Title/Abstract] OR "EpAbs"[Title/Abstract] OR "flebogamma*"[Title/Abstract] OR (("anticoronavirus"[Title/Abstract] OR "anticoronavirus"[Title/Abstract]) AND "immunoglobulin*"[Title/Abstract]))

\#9 (("anti-coronavirus"[Title/Abstract] OR "anticoronavirus"[Title/Abstract]) AND "immunoglobulin*"[Title/Abstract])

\#10 \#2 OR \#3 OR \#4 OR \#5 OR \#6 OR \#7 OR \#8 OR \#9

\#11 (("anti flu*"[Title/Abstract] OR "anti influenza*"[Title/Abstract] OR "antiflu*"[Title/Abstract] OR "antinfluenza*"[Title/Abstract]) AND ("plasma*"[Title/Abstract])) OR ("flu ivig*"[Title/Abstract])

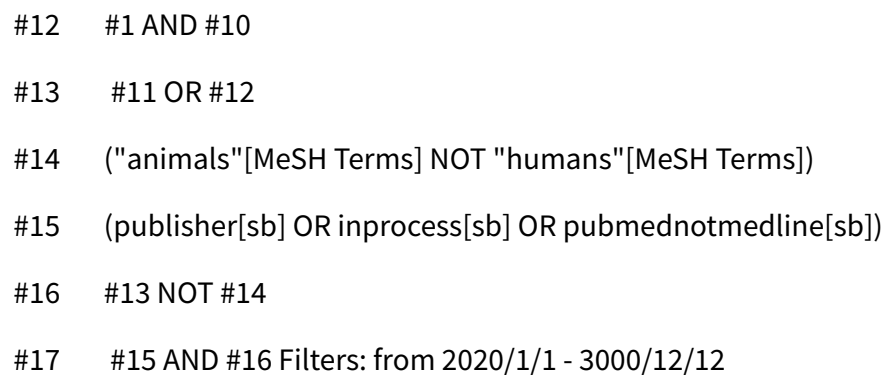

\section{Appendix 6. Search strategy - World Health Organization COVID-19 Global literature on coronavirus disease}

Advanced search; search fileds: title, abstract, subject

Search 1: (plasma OR convalesc* OR serum OR sera OR donor ${ }^{\star}$ OR donation* OR serotherapy OR "sero-therapy" OR "flu-IVIG" OR antiflu* OR "anti-flu" OR hyperimmune* OR hyper-immune* OR IVIG) AND (random* OR placebo OR RCT)

Search 2: (immunoglobulin OR immune-globulin OR globulin OR gamma-globulin OR $\gamma$-Globulin OR hyper-Ig) AND (random OR placebo OR RCT)

Search 3: ( immunization OR immunisation OR immunotherap*) AND (random* OR placebo OR RCT)

Search 4: ( CSL760 OR INM005 OR equine OR "XAV-19" OR "SAB-185" OR hIVIG) AND (random* OR placebo OR RCT)

Search 5: (INOSARS OR "GIGA-2050" OR "GIGA2050" OR "IGY-110" OR "IGY1109" OR GC5131 OR 5131A OR ITAC OR C-IVIG OR CIVIG OR flebogamma OR EpAbs) AND (random* OR placebo OR RCT) 


\section{Appendix 7. Search strategy - Epistemonikos, LOVE List Coronavirus disease (COVID-19)}

by pico search: population: Covid-19: intervention: passive immunization

Prevention or treatment: passive immunization: convalescent plasma: primary studies: RCTs

Prevention or treatment: passive immunization: Immunoglobulin therapy: primary studies: RCTs

Prevention or treatment: passive immunization: heterologous antibodies: primary studies: RCTs

\section{H I S T ORY}

\begin{tabular}{lll}
\hline Date & Event & Description \\
\hline 30 August 2020 & New search has been performed & $\begin{array}{l}\text { Two RCTs, eight controlled NRSIs and nine non-controlled NRSIs } \\
\text { included }\end{array}$ \\
\hline 30 August 2020 & $\begin{array}{l}\text { New citation required and conclusions } \\
\text { have changed }\end{array}$ & Additional safety data included (more than 20,000 participants) \\
\hline 3 June 2020 & $\begin{array}{l}\text { New citation required and conclusions } \\
\text { have changed }\end{array}$ & $\begin{array}{l}\text { We included results from one RCT and three controlled } \\
\text { NRSIs and added further safety data from non-controlled NRSIs. }\end{array}$ \\
\hline 31 May 2020 & New search has been performed & We included eight new studies. \\
\hline
\end{tabular}

\section{CONTRIBUTIONS OF AUTHORS}

CK: clinical expertise, study selection, data extraction and assessment, and conception and writing of the manuscript SJV: clinical expertise, study selection, data extraction and assessment, and conception and writing of the manuscript KLC: clinical expertise, study selection, data extraction and assessment, and conception and writing of the manuscript VP: methodological expertise, study selection, data extraction and assessment, and conception and writing of the manuscript $\mathrm{Cl}$ : methodological expertise, study selection, data extraction and assessment, and writing of the manuscript IM: development of the search strategy

EMW: clinical expertise and advice

AL: clinical expertise and advice

DJR: clinical expertise and advice

ZM: clinical expertise and advice

CS-O: clinical expertise and advice

LJE: clinical expertise, and conception and writing of the manuscript

NS: methodological expertise, study selection, data extraction and assessment, and conception and writing of the manuscript

\section{DECLARATIONS OF INTEREST}

CK: none known

SJV: is receiving a PhD scholarship from the not-for-profit Sanquin blood bank.

KLC: HSANZ Leukaemia Foundation PhD scholarship to support studies at Monash University. This is not related to the work in this review. VP: none known 
$\mathrm{Cl}$ : none known

IM: none known

EMW: I have received funding support from the Australian Medical Research Future Fund for a trial of convalescent plasma. I was not involved in bias assessment, data extraction or interpretation, but served as a content expert.

AL: none known

DJR: investigator on the REMAP-CAP and RECOVERY trial. I was not involved in bias assessment, data extraction or interpretation, but served as a content expert.

ZM: I have received funding support from the Australian Medical Research Future Fund for a trial of convalescent plasma. I was not involved in bias assessment, data extraction or interpretation, but served as a content expert.

CS-O: is a member of the BEST Collaborative Clinical Study Group and Associate Editor for Transfusion Medicine journal. I was not involved in bias assessment, data extraction or interpretation, but served as a content expert.

LJE: co-lead of the COVID-19 immunoglobulin domain of the REMAP-CAP trial and investigator on the RECOVERY trial. I was not involved in bias assessment, data extraction or interpretation, but served as a content expert.

NS: none known

\section{SOURCES OF SUPPORT}

\section{Internal sources}

- Sanquin Blood Supply, Netherlands Center for Clinical Transfusion Research

- University Hospital of Cologne, Germany Cochrane Cancer, Department I of Internal Medicine

- Monash University, Australia Transfusion Research Unit, Department of Epidemiology and Preventive Medicine

- NHS Blood and Transplant, UK NHS Blood and Transplant

- Leukaemia Foundation and HSANZ, Australia

Haematology Society of Australia and New Zealand (HSANZ)

\section{External sources}

- European Union's Horizon 2020 research and innovation programme, Belgium SUPPORTing high-quality evaluation of covid-19 convalescent plasma throughout EUROPE (Support-E)

\section{NOTES}

This review was previously part of 'Convalescent plasma or hyperimmune immunoglobulin for people with COVID-19: a living systematic review' (Piechotta 2021). The methodology for this review was developed concurrently with 'SARS-CoV-2-neutralising monoclonal antibodies for the treatment of COVID-19' (Kreuzberger 2021). 